\title{
PRELIMINARY RESULTS OF A DUST SCATTERING EXPERIMENT
}

\author{
P. BLIEK \\ P.L. LAMY \\ G. COURTES \\ Université de Provence, Marseille \\ Laboratoire d'Astronomie Spatiale, Marseille
}

\begin{abstract}
An experimental device, of the nephelometer type, to study the scattering properties of dust particles is presented. A fluidized bed generator produces a continuously flowing aerosol which is illuminated by either a He-Ne laser or a Xenon are lamp with interference filters. The size of the dust particles ranges between 1 and $30 \mu \mathrm{m}$. The scattering properties of dust particles are measured in the two directions of polarization. The first results are presented.
\end{abstract}

\section{EXPERIMENTAL PRINCIPLE AND SET-UP}

\subsection{Description}

The principle retained for our experimental investigation consists in generating a continuously flowing aerosol, a section of which is illuminated in order to study its scattering properties over the $0-180^{\circ}$ interval of scattering angles. It can therefore be considered as a nephelometer whose key part is a fluidized - bed generator which receives a mixture of the dust to be studied (in the form of powder) and of "large" glass spheres having diameters of 100 to $200 \mu \mathrm{m}$. An air flow forces the mixture to "boil"; the collisions between the glass spheres desagglomerate the powder and the liberated dust particles are transported by the air flow. The "boiling" chamber is fed by an endless screw which continuous1y enrichs the mixture so as to compensate for the loss of transported particles. It can be shown that the operation of the generator is governed by a very simple equation which related the ponderal concentrations of the mixture and the flow rates to the concentration of the aerosol. After a transitory phase of approximately $10 \mathrm{mn}$, the stationary regime given by the equation is reached and was experimentally shown to be remarkably stable. A nozzle concentrates the aerosol in a column having a diameter of $5 \mathrm{~mm}$; it is then allowed to flow freely over a length of $20 \mathrm{~mm}$ before being taken over by a suction device. The free part is of course used for the optical measurements. It is illuminated by a collimated beam from either an He-Ne laser or a Xenon arc-lamp. The optical part of the detection system is based an classic principles of photometry 
with two doublets and a fiels diaphragm (Fig. 1). A rotating polaroid polarizer is mounted at the entrance pupil. The scattered light is measured by a photomultiplier tube working in the analog mode. Two identical optical detection systems have been set up, one fixed to provide a reference so as to cancel any variation in the aerosol or in the illuminating souce and one mounted on a rotation table moved by a stepping motor. The whole experiment is under computer control. For a given scattering angle, we take the average of typically 3 measurements, each one having been previously divided by the corresponding reference signal.

\subsection{Characteristics}

The size range of dust particles allowed by the fluidized-bed generator depends upon the density of the dust but is typically 1 to $40 \mu \mathrm{m}$. The scattering volume has a diameter and an height of $5 \mathrm{~mm}$ and contains approsimately 100 to 1000 particles. Two illuminating sources are used:

i) a $7 \mathrm{~mW}$ Helium-Neon laser (6328 \&) with a beam expander yielding a useful diameter of $5 \mathrm{~mm}$;

ii) a $150 \mathrm{~W}$ Xenon arc lamp with an optical system giving a collimated beam having an internal angular dispersion of $\pm 2^{\circ}$ (given by the size of the arc) and interference filters centered at $4470,5500,6450,7060$, 7440 and $8240 \AA$ (typical bandpass $\sim 450 \AA$ ). The two detection systems have an angular aperture of $2^{\circ}$ and are equipped with Hamamatsu $R 378$ PMT whose sensitivity extends over the interval 1250-8500 \&. However, a cutoff at $3250 \AA$ is introduced by the lenses. Going further down in the u1tra-violet would simply requires changing the lenses and using a Xenon lamp with a quartz envelope. Finally, the mechanical set-up has been optimized to allow a range of scattering angles as large as possible, 5 to $174 \mathrm{deg}$.

\subsection{Main advantages}

The presence of a large number of grains in the scattering volume directly average the measurements over the various parameters characterizing them (e.g., roughness). The flow being turbulent, the average further extends over the randow orientations of the grains. The scattering light is also sufficiently intense to allow spectral measurements in a wide spectral domain with a source of reasonable power. The system has the capability to directly measure the volume scattering function for a dust population having a selected size distribution. However the main drawback is the absolute calibration which requires the exact determination of the concentration in the aerosol. Although the required equipment is commercially avaible, we did not have yet the possibility to acquire it.

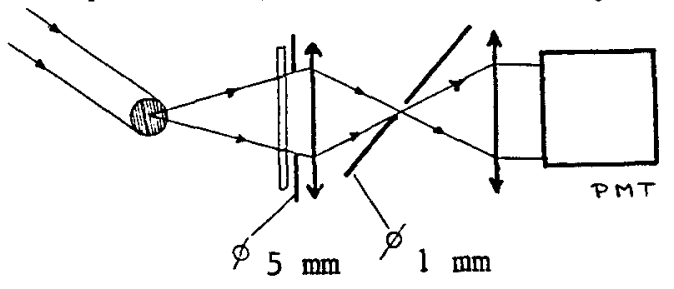

Fig. 1.: Optical layout of the detection system 

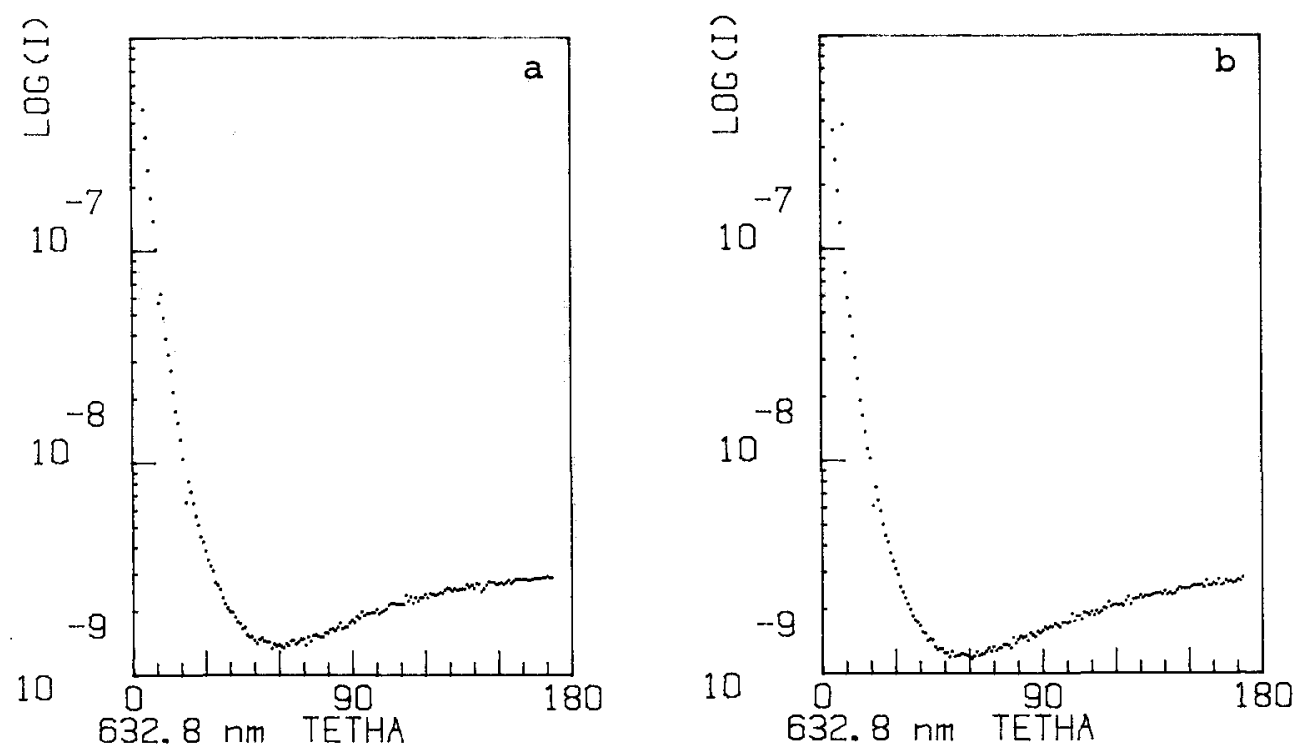

Fig. 2 : Total intensity scattered by iron grains under He Ne laser illumination as a function of scattering angle. This illustrates the stability of the measurements as the two results $a$ and $b$ were obtained at a one day interval.

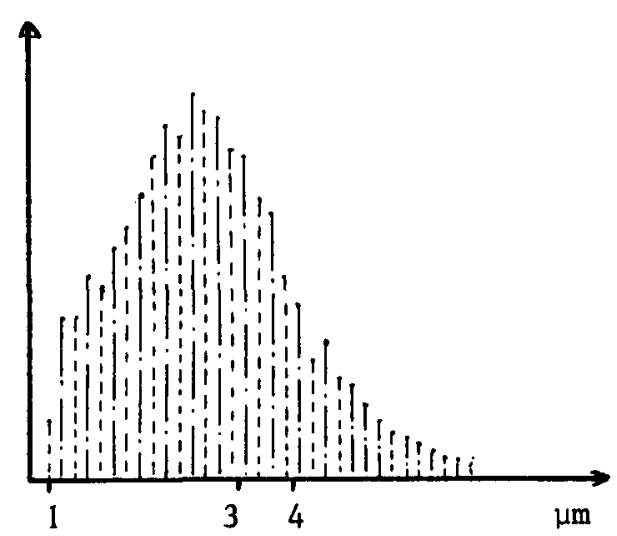

Fig. 3: The size distribution function of silicon dioxyde grains

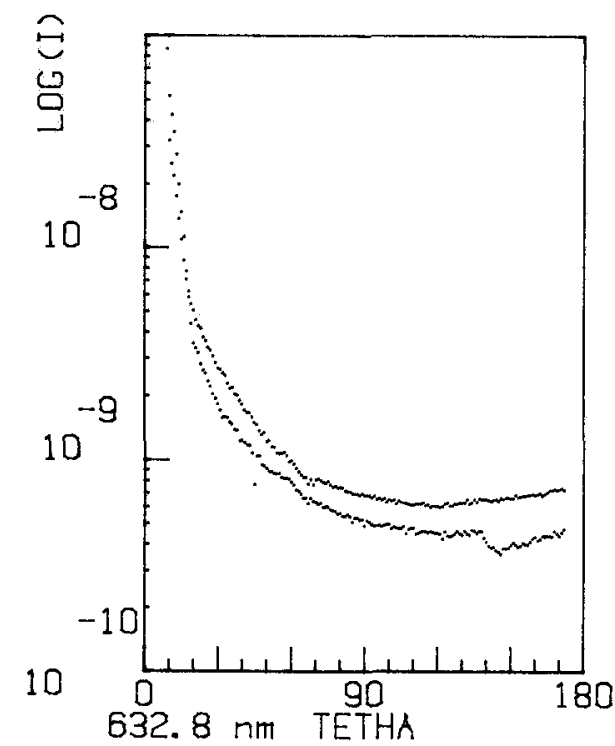

Fig. 4 : The polarized intensities scattered by silicon dioxyde grains under He Ne laser illumination 


\section{PRELIMINARY RESULTS}

The results presented here were obtained with commercially available powders and with the He-Ne laser. Fig. 2 is intended to demonstrate the reproductibility of the measurements: the same iron powder was used for two different runs at a one-day interval. The difference is well within the error bars as given by the dispersion of the data points. This proves that the method of normalization using the reference signal is working very well. A silicon dioxyde powder was also studied. Its size distribution was measured independently with a Coulter counter and peaks at a diameter of $2.33 \mu \mathrm{m}$ (Fig. 3). The silicon dioxyde aerosol was illuminated by He-Ne laser light polarized in the parallel and perpendicular directions w.r.t. the scattering plane. The results appear in Fig. 4: the upper curve corresponds to the parallel case and the lower one, to the perpendicular case. However, they have been obtained with two different runs and therefore, should not be compared on an absolute basis. The qualitative behaviour is nevertheless typical of dielectric micronic grains (Giese, 1971). Several tests have been performed with the Xenon $1 \mathrm{amp}$ and results for different wavelengths will hopefully be presented in the near future.

\section{REFERENCE}

Giese, R.H.: 1971, Tabellen Von Mie - Streu Funktionen I. Gemische dielektrischer Teilchen, BMBW-FB W 71-23 Ruhr-Universität Bochum 